\title{
Surgical treatment of cerebral cysticercosis: long-term results and prognostic factors
}

\author{
Benedicto O. Colli, M.D., Ph.D., Carlos G. Carlotti, Jr., M.D., Ph.D., \\ João A. Assirati, JR., M.D., Hélio R. Machado, M.D., Ph.D., \\ Marcelo Valença, M.D., Ph.D., and Marcelo C. M. Amato, M.C.M. \\ Division of Neurosurgery, Department of Surgery, Ribeirão Preto Medical School, University of São \\ Paulo, Ribeirão Preto, São Paulo, Brazil
}

\begin{abstract}
Object. Cysticercosis is the most frequent parasitosis of the central nervous system. Although anticysticercal drugs have proven efficient in some cases, many patients with NCC require palliative, occasionally curative, surgical procedures. The authors analyzed the data and prognostic factors obtained during the follow-up period (range 1-255 months, median 38 months) in 160 patients with cerebral cysticercosis who underwent surgical treatment.

Methods. Different surgical approaches were indicated to control increased intracranial pressure (ICP) in most patients, and some patients had undergone decompressive surgery for local brain lesions. Most patients required more than one surgical procedure. Statistical analysis was performed using the Fisher exact, the log-rank, and the Kruskall-Wallis tests. Survival curves were calculated according the Kaplan-Meier method. The removal of a giant cyst from the parenchyma or cisterns for relief of increased ICP and for chiasm/optic nerve decompression improved most symptoms in patients. The removal of ventricular cysts was effective in the control of increased ICP in most patients. Patients with a ventricular cyst and ependymitis/arachnoiditis required placement of a ventriculoperitoneal (VP) shunt after the cyst was removed. This therapy effectively controlled increased ICP. Patients younger than 40 years of age at the time of treatment and male patients had worse outcomes. The outcome in patients who underwent VP shunt surgery or shunt surgery combined with reservoir implantation was worse than that in those who underwent cyst removal alone. Shunt-related infection was the most frequent complication, and the global mortality rate during the follow-up period was $21.2 \%$. Although both complications were more frequent in the first 2 postoperative years, they occurred at any time.

Conclusions. Long-term prognosis in patients with cerebral cysticercosis who required surgery was not good. Cysts located in the basal cisterns and patient age younger than 40 years were poor prognostic factors
\end{abstract}

\section{KEY WORDS - cysticercosis • intracerebral cyst • hydrocephalus • intracranial hypertension $\quad$ ventriculoperitoneal shunt}

Cysticercosis of the CNS is an infestation with Cysticercus cellulosae, the larval form of Taenia solium. Because of migration, infection is worldwide and represents the most frequent parasitosis of the CNS. As a transmissible disease, its high incidence reflects the poor basic sanitation conditions of a population, and it should be considered a hallmark of underdevelopment. ${ }^{14}$ Humans are usually the definitive host of $T$. solium, which lives in the intestines. The proglottids of the parasite are passed with human feces and break open in the external medium, releasing a high number of eggs. Pigs normally ingest the eggs; in the acid and enzymatic conditions of the stomach, the embryophore dissolves and the oncosphere, by the ac-

Abbreviations used in this paper: $\mathrm{CNS}=$ central nervous system; $\mathrm{CSF}=$ cerebrospinal fluid; $\mathrm{CT}=$ computerized tomography; ICP = intracranial pressure; $\mathrm{MR}=$ magnetic resonance; $\mathrm{NCC}=$ neurocysticercosis; $\mathrm{PFC}=$ posterior fossa craniectomy; $\mathrm{SD}=$ standard deviation; $\mathrm{TCA}=$ transcallosal approach; $\mathrm{VP}=$ ventriculoperitoneal. tion of the bile and digestive enzymes, is activated and liberated after tearing the oncospheral membrane. The hexacanthous embryo invades the intestinal mucosa, enters the lymphatic or blood circulation, and finally establishes itself mainly in the CNS, muscles, subcutaneous tissue, and eyes. The embryos develop into a metacestode called C. cellulosae, which is a cystic organism composed of a complex wall surrounding a cavity that contains vesicular fluid and a scolex. Humans acquire T. solium by ingestion of pork infested with $C$. cellulosae. In the digestive tract the metacestode finds suitable conditions to evaginate and develop into the adult tapeworm, thus completing its natural evolutionary cycle. Humans acquire cysticercosis when infested with $C$. cellulosae. Infestation may occur by self-infestation (with eggs eliminated by the intestine of the individual himself) or by heteroinfestation (ingestion of vegetables or water contaminated with eggs passed by other persons), which represents the most important epidemiological form by which the disease is acquired. 
In developing countries, NCC is important not only because of its frequency but also because of high morbidity and mortality rates associated with it, especially in cases in which it progresses to increased ICP. ${ }^{17,18}$ In our region, NCC has been observed in 2.7 to $7.5 \%$ of patients hospitalized with neurological dysfunction. ${ }^{1,58}$ In our hospital $35.6 \%$ of these patients presented with increased ICP. ${ }^{58}$

Despite the development of medications, such as praziquantel and albendazole, that can provoke degeneration of cysticerci, recent experience $e^{2,12,27,28,44,49,51,52,54,57,59,62}$ has shown that their efficacy is more marked in cases in which active cysts are present in the brain parenchyma. Their effect on ventricular or cisternal cysts has been report$\mathrm{ed},{ }^{27,28,44}$ but there is no consensus regarding their efficacy in these situations. Furthermore, the use of these medications does not prevent the occurrence of complications such as hydrocephalus requiring surgical treatment. For this reason, a considerable number of patients with NCC require surgical procedures that are usually palliative but may sometimes be curative.

The present report is based on the 22-year experience of the Division of Neurosurgery of the Hospital das Clínicas, Ribeirão Preto Medical School, University of São Paulo, in the surgical treatment of patients with NCC as well as on review of the pertinent literature.

\section{Pathological Anatomy}

The hexacanthous embryo reaches the CNS, particularly through the bloodstream, stopping in the brain parenchyma by occluding a capillary. After actively crossing the capillary wall, the embryo reaches the interstitial space where it evolves to the cystic form, transforming into $C$. cellulosae. ${ }^{30}$ The infestation of the ventricles is attributed to active passage of hexacanthous embryo through the capillaries of the choroid plexus ${ }^{30}$ immediately followed by their transformation into cysticerci. Within the ventricles, depending on its size, the cysticercus is carried by the CSF to the subarachnoid space. Controversy exists regarding the direct arrival of the hexacanthous embryo in the subarachnoid space or the ventricles through meningeal or subpial vessels. ${ }^{30}$ After becoming established in the CNS, C. cellulosae begins to grow, usually reaching 4 to $20 \mathrm{~mm}$ in widest diameter when located inside the brain parenchyma. The cysticerci developing in the ventricles or in the subarachnoid space usually reach a larger size and often take on the form of $C$. racemosus, which is characterized by a membrane of irregular thickness, the absence of a scolex, and usually being clustered in multiple vesicles generally interconnected, resembling a raceme. Although racemose cysts are frequently observed in ventricles and cisternae, they may also occur in the brain parenchyma. ${ }^{20}$

The cysticerci present a natural evolutionary process that culminates with their degeneration within a period of approximately 2 to 5 years. ${ }^{30}$ This process starts after the transformation of the hexacanthous embryo into the cysticercus (live or active cyst [vesicular stage]), followed by a stage in which the cyst begins to undergo degenerative changes characterized by thickening of the membrane and replacement of the clear fluid with a whitish gel (degenerating cyst [colloidal stage]); with the progression of this process the wall continues to become thicker and the gel undergoes calcium deposition (granular stage), and finally the cyst becomes completely calcified, being reduced to one third or one fourth of its original size (calcified nodular stage).

The host's reaction to the cysticercus ${ }^{30,53}$ is characterized by an inflammatory process around the parasite, similar to a granulomatous reaction to a foreign body. The inflammatory reaction to the cysticercus is related to the phase of cyst development and is less intense during the cystic or active phase, becomes more marked when the cyst starts to degenerate, and progressively declines until the cyst becomes calcified. The intensity of the inflammatory reaction depends on the parasite-host interaction in the nervous system..$^{30,53}$ The inflammatory reaction triggered by cysts in direct contact with CSF of the ventricles and subarachnoid space may extend to other sites and provoke extensive areas of inflammation. During this process, clinical manifestations become acute and increased numbers of cells and higher protein levels, as well as the presence of immunodiagnostic criteria, are found in the CSF.

\section{CLINICAL MATERIAL AND METHODS}

\section{Patient Population}

In this study we analyzed data obtained in 160 consecutive patients with cerebral cysticercosis who underwent surgery performed primarily by the same surgeon (B.O.C.) in the Division of Neurosurgery, Hospital das Clínicas, Ribeirão Preto Medical School, University of São Paulo, between January 1980 and December 2001. Indications for surgery were to control elevated ICP, to remove cysts causing local compression, and, rarely, to obtain diagnosis. The clinical courses of these patients were retrospectively and/or prospectively analyzed. Treatment and results for some of these patients have been previously reported..$^{17,18}$ In this article we focus on the entire group and analyze the long-term follow-up data and the prognostic factors obtained in these patients.

\section{Clinical Features and Examinations}

Diagnosis of cerebral cysticercosis was made by undertaking CSF evaluation and/or neuroradiological examinations (ventriculography, CT scanning, CT cisternography, CT ventriculography, and MR imaging of the skull), intraoperative evaluation, and histopathological examination. One or more CSF samples were studied during different periods of the disease in 138 patients (86.3\%). Pleocytosis with more than $2 \%$ eosinophils ${ }^{81}$ was found in 12 patients (7.5\%); antigen/antibody tests (complement fixation test, indirect immunofluorescence test, and enzyme-linked immunosorbent assay) were positive in 57 (35.6\%) patients; and eosinophils and positive antigen/antibody tests were found in 31 patients $(19.4 \%)$. The neuroradiological criteria for the diagnosis of NCC were the presence of two or more calcifications on CT scanning, two or more cysts on CT scanning, images suggesting cysts on ventriculography/CT ventriculography, inflammatory obstruction of the aqueduct or fourth ventricle on ventriculography in adult patients coming from an endemic area, and identification of cysts on MR imaging (Figs. 1-4). Diagnosis was deter- 
Surgical treatment of cerebral cysticercosis

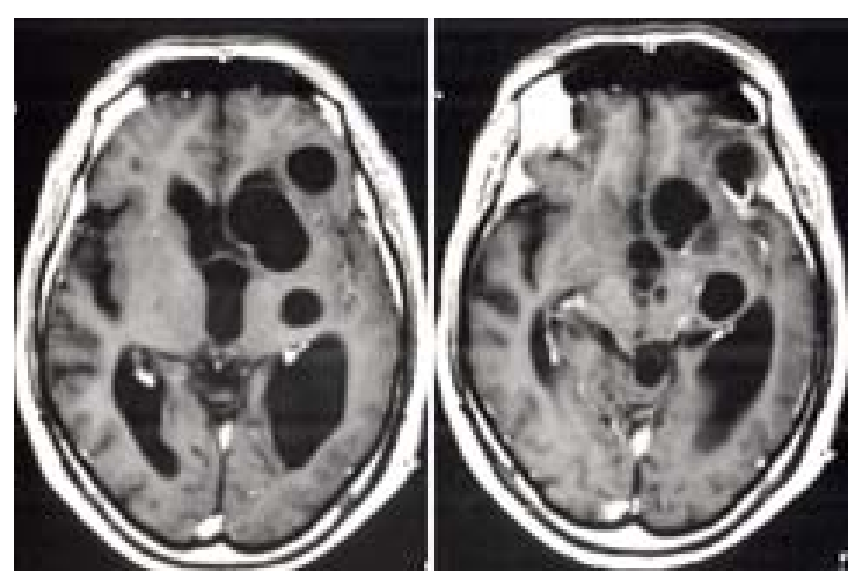

Fig. 1. Axial MR images. Noncontrast (left) and contrast-enhanced $\mathrm{T}_{1}$-weighted (right) images of the brain revealing several cysts in the parenchyma, cisterns (prepontine and lamina quadrigeminal) and lateral ventricle. The cysts are compressing and distorting the brainstem, and a degenerating cyst is found in the right frontal lobe (right).

mined based on CSF changes in 10 patients (6.3\%), neuroradiological features in 16 patients $(10 \%)$, CSF changes and neuroradiological features in 41 patients $(25.6 \%)$, neuroradiological features and intraoperative findings (Figs. 1,2 , and 4 ) in 38 patients (23.7\%), and on CSF changes, neuroradiological features and intraoperative findings in 55 patients (34.4\%). Diagnosis was confirmed at autopsy in five patients. Thirteen patients had previously undergone placement of a VP shunt at another institution.

Patient age at the onset of the disease ranged from 11 to 70 years; 117 patients $(73.1 \%)$ were 20 to 50 years of age and $82(51.3 \%)$ were 20 to 40 years old (mean $36.7 \pm$ 13.9 [ $\pm \mathrm{SD}$ ] years, median 35 years). Eighty-six patients $(53.8 \%)$ were male and 74 were female $(46.2 \%)$ (male/ female ratio 1.16:1). There was no significant age difference between males and females $(\mathrm{p}=0.5760$, $\mathrm{df}=1$ [Fisher exact test]).

Duration of symptoms on admission ranged from 1.5 hours to 17 years $(81.9 \%$ of patients had experienced symptoms for $<1$ year; $73.8 \%$ for $<6$ months; $38.1 \%$ for $<1$ month; and $9.4 \%$ for $<1$ day). Clinical manifestations at the onset of disease are summarized in Table 1. The most common signs and symptoms were those caused by intracranial hypertension followed by gait disturbance, seizures, and dizziness/vertigo. Signs of increased ICP were present in 155 patients $(96.9 \%)$ and intracranial hypertension was caused by hydrocephalus in 134 patients $(86.5 \%)$, cerebral edema (cysticercal encephalitis or pseudotumoral form) in one patient $(0.6 \%)$, giant cysts behaving as expansive lesions (tumoral form) in $12(7.7 \%)$, and combined giant cysts and hydrocephalus in eight (5.2\%), for a total of 142 patients (91.6\%) with hydrocephalus and $20(12.9 \%)$ with the tumoral form. Ten remaining patients exhibited signs of local compression (five with hemiparesis and/or seizures and five with optoquiasmatic compression). Five patients also presented with signs of spinal cord, radicular, or cauda equina compression associated with signs of intracranial involvement. Patients with spinal cord/radicular/cauda equina signs in whom there was

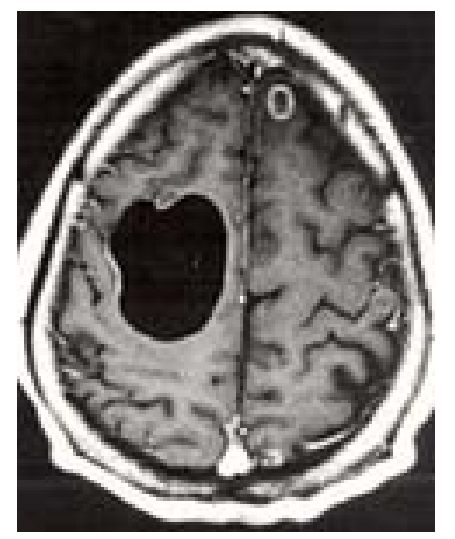

Fig. 2. Axial contrast-enhanced $\mathrm{T}_{1}$-weighted $\mathrm{MR}$ image of the brain obtained in a patient with progressive right hemiparesis, revealing a giant cyst starting to degenerate (contrast enhancement in its wall) in the left parietal region; some mass effect on the adjacent gyrus and a degenerating cyst in the right frontal pole can be seen.

no clinical evidence of brain disease were excluded from this review. All patients except those in whom precocious death occurred attended follow-up examinations for at least 1 month at our institution after discharge from the hospital. The patients were followed at intervals of approximately 6 months.

Computerized tomography of the skull was performed in all patients at least one time during the follow-up period, and it confirmed the diagnosis in 89 cases (those with calcifications and hypodense, enhanced, and annular enhanced nodules [cyst in different phases of evolution], and giant cysts). Additionally, in several patients CT scanning demonstrated hydrocephalus and rounded deformations of the ventricles, suggesting the presence of cysts, a fact that was not always confirmed by ventriculography. Contrast enhancement along the wall of the fourth ventricle was observed in only four patients, suggesting the presence of degenerating cysts adhering to the ventricular wall, as confirmed intraoperatively.

Magnetic resonance imaging of the brain was performed in 20 patients and, in addition to hydrocephalus, it revealed identification of parenchymal and cisternal cysts in several cases as well as direct (Fig. 2) or indirect evidence of fourth ventricular cysts in other cases (observed as a rounded dilation of this cavity).

When CT and/or MR imaging indirectly suggested the presence of a cyst within a ventricle or the basal cisterns, ventriculography, CT ventriculography, and/or CT cisternography was performed. Ventriculography confirmed the diagnosis of intraventricular cysts in 30 cases (Fig. 4) and CT ventriculography in 10 , and CT cisternography disclosed cisternal cysts and/or fourth ventricle cysts in 10 patients (Figs. 1 and 3).

\section{Disease Management}

Medical Treatment. Thirty-six patients received anticysticercal drugs (praziquantel and albendazole in 18 cases each). Twenty-two patients underwent medical therapy before surgery and 14 after surgery. In eight patients only hydrocephalus was found on CT scanning before 


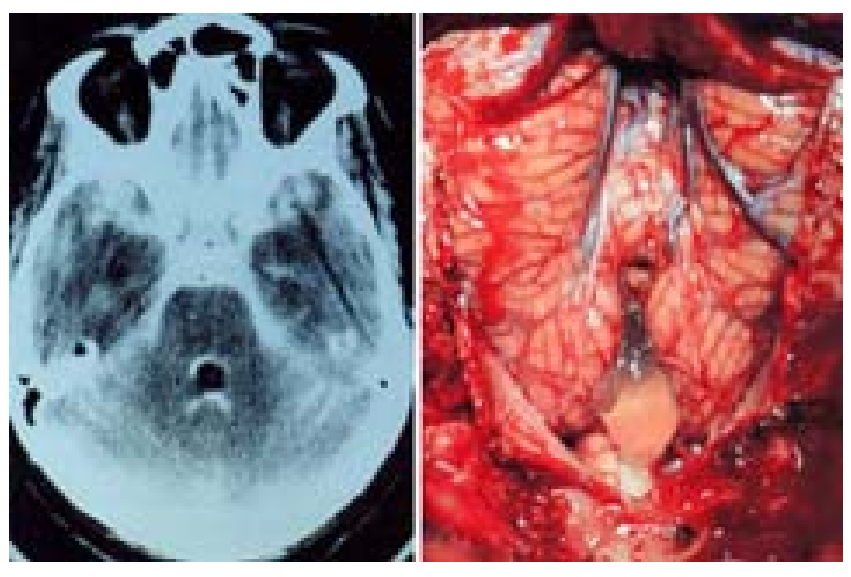

Fig. 3. Images obtained in a patient with intermittent increased ICP. Left: A CT cisternography scan demonstrating a cysticercus in the fourth ventricle delineated by contrast. Right: Intraoperative photograph of the posterior fossa obtained with the patient in the sitting position. A free active cysticercal cyst descends from the foramen of Magendie. Note that the cyst membrane is transparent, and its fluid content is colorless, and there is no associated arachnoiditis.

medical treatment and in one only edema was observed (pseudotumoral form). The remaining 27 patients harbored cysts in the parenchyma, ventricles, and/or cisterns (23 with parenchymal cysts, 16 with cisternal cysts, and five with ventricular cysts). Most of these patients were treated by Dr. Takaynagui, and the results of the medical treatment of patients with NCC have been already reported..$^{57,59}$

Surgical Treatment. Surgical treatment was performed to control increased ICP in 135 patients, to treat intracranial hypertension and local brain compression in 20, and to treat local brain compression alone in five. Increased ICP was caused by hydrocephalus in 134 (83.8\%) of 155 patients, intracranial space-occupying lesion (tumoral form) in $12(7.7 \%)$, hydrocephalus and giant cysts in eight (5.25\%), and pseudotumor cerebri (pseudotumoral form) in one case. Based on the pathophysiological mechanisms of intracranial hypertension detected on conventional CT scanning, ventriculography, CT cisternography, CT ventriculography, and/or MR imaging, different surgical approaches were indicated. In patients harboring tumoral lesions a direct approach was performed and the cyst removed; in those with pseudotumoral form refractory to clinical treatment for increased ICP, we undertook decompressive craniectomy and direct removal of ventricular/ cisternal cysts and/or placed a VP shunt in those with hydrocephalus. Fifteen patients had undergone 16 previous operations elsewhere (15 VP shunt surgeries and one posterior fossa craniectomy), and 248 surgical procedures (241 in 145 patients) were performed at our institution. Fifty-one patients underwent one procedure, 55 two, 26 three, and 28 underwent four or more surgical procedures; a total of 280 surgeries were performed in the 160 patients (1.75 procedures/patient). Operations included the implantation of 68 reservoirs for transient control of increased ICP or to perform ventriculography/CT ventriculography (57 as the first procedure and 11 as an additional

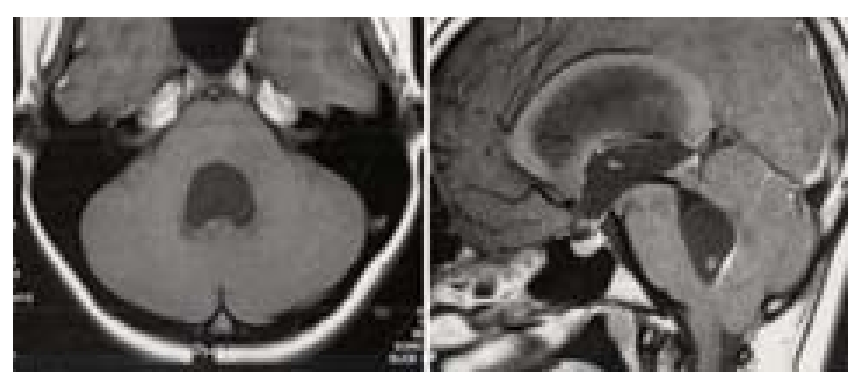

Fig. 4. Contrast-enhanced axial (left) and sagittal (right) $\mathrm{T}_{1}$ weighted MR images of the brain. The fourth ventricle is enlarged and the membrane rounded. The scolex of the cyst is seen as an area of increased signal intensity in the inferior part of the ventricle (right).

procedure); $105 \mathrm{VP}$ shunt procedures (56 as the first procedure and 49 as an additional procedure); 49 PFCs for exploration of the basal cisterns and/or fourth ventricle (11 as the first procedure and 38 as an additional procedure); 25 supratentorial craniotomies for removal of cisternal cysts (five causing compression of the optic nerve/chiasm and 12 giant cysts causing intracranial hypertension) and for eight giant parenchymal cysts (22 as the first procedure and three as an additional procedure); 17 TCA procedures to the interhemispheric fissure and to the third and/or lateral ventricles for cyst removal (seven as the first procedure and 10 as an additional procedure); nine endoscopic approaches to the ventricles for cysts removal (six as the first procedure and three as an additional procedure); three laminectomies as additional procedures; two hypothalamic ventriculostomies with catheter placement (one as the first procedure and one as an additional procedure); one subfrontal craniotomy to repair a CSF leak (first procedure); and one bilateral temporal decompressive craniotomy (first procedure).

Patients who underwent surgery for the control of increased ICP were classified as improved when there was full remission of clinical signs of increased ICP; un-

TABLE 1

Summary of the most frequent initial signs and symptoms in 160 patients with cerebral cysticercosis

\begin{tabular}{lc}
\hline \hline Signs and Symptoms & $\begin{array}{c}\text { No. of } \\
\text { Patients }(\%)\end{array}$ \\
\hline headache & $119(74.4)$ \\
nausea/vomiting & $75(46.9)$ \\
papilledema & $72(45.0)$ \\
gait disturbance & $41(25.6)$ \\
hemiparesis & $13(8.1)$ \\
crural paraparesis & $6(3.8)$ \\
quadriparesis & $3(1.9)$ \\
pyramidal signs & $6(3.8)$ \\
seizures & $36(22.5)$ \\
dizziness/vertigo & $35(21.9)$ \\
blurred vision & $25(15.6)$ \\
decreased vision & $14(8.8)$ \\
somnolence & $21(13.1)$ \\
loss of consciosuness & $10(6.3)$ \\
mental confusion & $18(11.3)$ \\
neck stiffness & $17(10.6)$ \\
\hline
\end{tabular}


changed when signs of intracranial hypertension persisted despite surgery; and worsened when the surgical procedure or its complications resulted in worsening of the clinical picture or in death. Several patients who were considered improved at final evaluation required one or more shunt revisions and others continued to experience disease-related neurological sequelae, usually some form of visual dysfunction.

Patients in whom the cyst was excised in the opticochiasmatic region underwent subjective and/or objective examinations to determine visual function pre- and postoperatively and were classified as improved or unchanged.

Patients in whom intraventricular or parenchymal giant cysts were excised underwent evaluation for signs of increase ICP and for the course of the clinical signs resulting from the lesion's local compression and mass effect.

The quality of survival was considered good when the patient was able to return to work, moderate when he was capable of performing his daily activities, and poor when he was confined to his bed. Surgery-related mortality was considered to be early when death occurred during the first 30 days postoperatively, and "late" when it occurred after the 30th postoperative day.

\section{Statistical Analysis}

Statistical analysis was performed using the Fisher exact test for comparing proportions and log-rank test, and Kruskall-Wallis nonparametric analysis of variance (with the Dunn multiple comparisons posttest) for comparison of survival curves and rates. An $\alpha$-error value not exceeding $5 \%$ was considered significant for the two-tailed tests. The tests were performed using the GraphPad PRISM (version 2.0; GraphPad Software, Inc., San Diego, CA).

\section{RESULTS}

\section{Medical Treatment}

In $18(78.2 \%)$ of the patients with parenchymal cysts (three [18.8\%] with cisternal cysts, and one [20\%] with ventricular cysts), the cysts disappeared or became calcified after the anticysticercal therapy. Nevertheless, in the patients with cisternal and ventricular cysts there was doubt whether the resolution was an effect of the natural history or a response to the drugs, because the time period between treatment and disappearance of the cyst was longer than 6 months in all patients. Seventeen of 22 patients who underwent preoperative anticysticercal therapy developed intracranial hypertension that required some surgical treatment during the follow-up period. One patient with the pseudotumoral form of NCC died of complications related to decompensation of intracranial hypertension after treatment with praziquantel, despite undergoing bilateral temporal decompressive craniectomies.

\section{Surgical Treatment}

In two patients who underwent hypothalamic ventriculostomy with catheter placement (follow-up duration 184 and 263 months, respectively) and two patients who underwent reservoir implantation (follow-up duration 180 and 192 months, respectively), increased ICP resolved, and the patients required no other treatment. In all other patients in whom a reservoir was implanted, the procedure was undertaken to perform ventriculography/CT ventriculography or to obtain transient relief of the increased ICP. A bilateral temporal decompressive craniotomy was performed in one patient with diffuse infestation of the cerebral parenchyma and edema causing refractory increased ICP; however, the patient did not improve and died a few days after surgery.

Seventeen patients underwent pterional craniotomies for cisternal cyst removal (five causing compression of the optic nerve/chiasm and 12 giant cysts causing intracranial hypertension and/or local brain compression); their follow-up course ranged from 1 to 168 months (mean $84.7 \pm$ 53.6 months $[ \pm \mathrm{SD}]$, median 89 months). In three patients with compression of the optic nerve/chiasm, the visual deficit improved in one, stabilized (unchanged), and in another the visual deficit worsened (follow-up period 6 168 months). Eleven of 12 patients in whom pterional craniotomy was performed to remove cisternal cysts causing increased ICP improved, and one died.

Eight patients underwent excision of giant parenchymal cysts causing increased ICP and/or local brain compression. Seven improved in a follow-up period of 1 to 231 months (mean $92.9 \pm 77.9$ [SD], median 110.5 months). The eighth patient, who was comatose, died.

In 17 patients a TCA was performed to resect third/lateral ventricular cysts, and the follow-up period was 1 to 255 months (113.6 \pm 79.8 months, median 96 months). The cyst was found in the third and lateral ventricles in 11 patients; cysts and ependymitis in four patients; and an additional cyst in one of these patients found in the fourth ventricle was removed via the same approach (a catheter was placed and the cyst was evacuated through the cerebral aqueduct). In one patient a cyst was present in the interhemispheric fissures, and in another patient only ependymitis was found. In eight patients (72.7\%) who harbored only ventricular cysts and who did not previously undergo VP shunt placement increased ICP improved. The two patients with cyst and ependymitis - in whom no previous shunt surgery had been performed-required this therapy after undergoing surgery via the TCA.

In eight patients an endoscopic resection of ventricular cysts was conducted (three were excised in the fourth ventricle and four in the lateral/third ventricles); endoscopy was also performed to treat inflammatory adherence (one from the lateral ventricles). The follow-up course in these eight patients ranged from 5 to 85 months (mean $37 \pm$ 34.1 months, median 27.5 months). Removal of cysts from the fourth ventricles was achieved in two patients, and the remaining patient required a PFC because the cyst could not be identified endoscopically. Removal of the cysts from the lateral/third ventricles was successful in three patients and in two patients a TCA was required (because the cyst could not be identified in one patient and because of venous bleeding in the other).

Fifty-two PFCs with or without cyst excision were performed in 49 patients (as first surgery in 11, after reservoir implantation in 23, after VP shunt placement and/or surgical procedure other than reservoir implantation in 18 , and after endoscopic approach to the fourth ventricle in one patient); the follow-up period was 1 to 237 months (mean $98.2 \pm 65.6$ months, median 101 months). Of the 30 patients who initially underwent PFC or reservoir 
implantation followed by PFC, free cysts were present in 19 patients without or with mild fourth ventricular ependymitis, signs of increased ICP improved in 13 patients (68.4\%), and five patients required a VP shunt 1 to 4 months later. In nine patients we found degenerating cysts and ependymitis/arachnoiditis in the fourth ventricle and/or in the basal cisterns of the posterior fossa, of whom three $(66.7 \%)$ required a VP shunt from 1 day to 6 months after the PFC. In two patients free cysts were presented in the fourth ventricle and in basal cisterns of the posterior fossa and they required a VP shunt, respectively, 5 days and 1 month after the PFC. One $(2.6 \%)$ of the 39 patients who underwent PFC, with inflammatory obstruction of the fourth ventricle, fourth ventricular degenerating free cyst, and degenerating cysts around the medulla oblongata adherent to the nervous structures, suffered neurological worsening.

Fifty-six patients underwent VP shunt placement as first surgery to control increased ICP and 49 underwent this procedure after previous surgical interventions that failed to control increased ICP; their follow-up period was 1 to 255 months (mean $65.5 \pm 63.5$ months, median 44 months).

In $53(50.5 \%)$ of 105 patients who underwent VP shunt surgery one or more shunt revisions were required, for a total of 164 procedures (1.56 procedures/patient). Shunt revisions were required secondary to shunt malfunctioning in 126 cases (76.8\%); indications included problems with the proximal catheter in 46, problems with the distal catheter in 13, excessive drainage in six, and nonspecified malfunction in 61. Revision secondary to infection was required in 38 cases $(23.2 \%)$.

\section{Follow-Up Review}

The overall clinical follow-up period ranged from 1 to 255 months (mean $46.1 \pm 40.2$, median 38 months).

Survival. The 5-, 10-, and 20-year survival estimates were $88.8,84.7$, and $52.4 \%$, respectively.

The survival estimates for patients with cerebral cysticercosis according to their age at diagnosis were 88.6 and $75.4 \%$ at 5 years; 88.6 and $69.6 \%$ at 10 years; and 78.0 and $52.2 \%$ at 20 years, respectively, for patients who were 40 years of age or younger and patients who were older than 40 years of age. There was significant difference between the curves for the two age groups ( $p=$ $0.0217, \mathrm{df}=1$ [log-rank test]).

The survival estimates according to distribution of sex were 73.8 and $86.4 \%$ at 5 years; 64.6 and $86.4 \%$ at 10 years; and 60.7 and $81.8 \%$ at 20 years for female and male patients, respectively. There was significant intergroup difference $(\mathrm{p}=0.0167, \mathrm{df}=1$ [log-rank test]). Comparison of survival curves for patients who underwent VP shunt surgery and those who underwent reservoir placement combined with VP shunt surgery showed similar results: that is, a worse prognosis for males than females ( $\mathrm{p}=$ $0.032, \mathrm{df}=1$ [log-rank test]). The survival estimates were 62.8 and $90.3 \%$ at 5 years; 50.2 and $90.3 \%$ at 10 years; and 50.2 and $90.3 \%$ at 20 years for female and male patients, respectively.

Survival curves were determined for patients with NCC who underwent 1 ) shunt surgery and shunt surgery combined with reservoir implantation, 2) PFC for removal of posterior fossa/fourth ventricular cysts, 3) TCA to the third/fourth ventricles for cyst removal, and 4) resection of cisternal cyst, performed as single surgical procedures. The 5-year survival estimates were 80.4, 94.4, 100, and $90 \%$; the 10-year survival estimates were $80.4,94.4,87.5$, and 90\%; and the 20-year survival estimates were 73.7, 94.4, 87.5, and 90\% for patients in whom we performed shunt surgery, PFC, TCA, and cisternal cyst resection, respectively. Significant differences were demonstrated ( $p<0.001$, [Kruskall-Wallis nonparametric analysis of variance]). Posttest analysis (the Dunn multiple comparisons test) showed significant differences among the survival curves for patients who underwent VP shunt surgery and PFC $(p<0.001)$; those who underwent VP shunt surgery and TCA $(p<0.001)$; those who underwent VP shunt surgery and cisternal cyst resection $(\mathrm{p}<0.001)$; those who underwent PFC and TCA $(p<0.001)$; and those who underwent PFC and cisternal cyst resection. No significant difference, however, was shown between survival curves for patients who underwent TCA and those who underwent cisternal cyst resection.

\section{Postoperative Functional Outcome}

We studied the evolution of clinical signs of intracranial hypertension in the patients in whom VP shunts were placed. Of patients followed for 2 or more years, in most intracranial hypertension was absent by 2 years, although some required one or more shunt revisions. Despite good control of ICP four patients (33.3\%) died 2 years postoperatively.

The quality of survival in this series of patients is summarized in Table 2. Of 34 patients who died during the overall follow-up period, 21 deaths $(61.8 \%)$ occurred in the first 2 postoperative years and $13(38.2 \%)$ occurred after this period.

TABLE 2

Quality of survival in all patients treated surgically

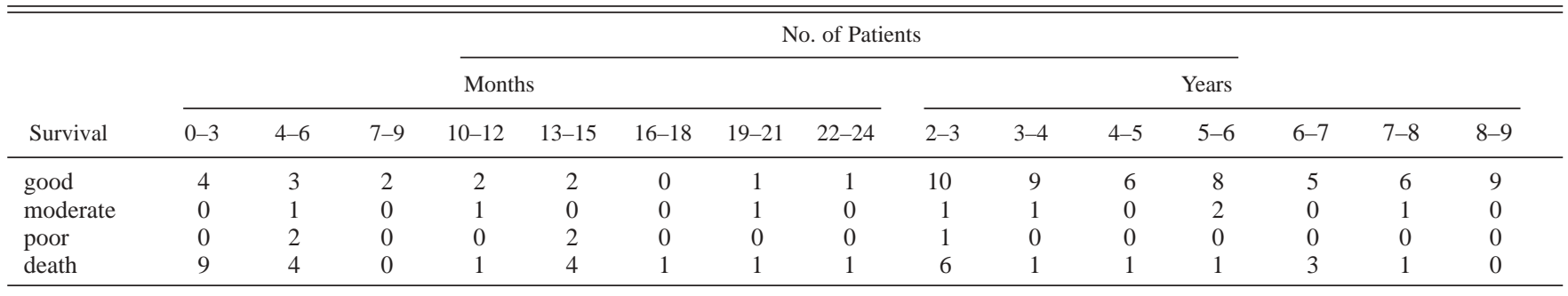


TABLE 2, continued

Quality of survival in all patients treated surgically

\begin{tabular}{|c|c|c|c|c|c|c|c|c|c|c|c|c|c|}
\hline \multirow[b]{2}{*}{ Survival } & \multicolumn{13}{|c|}{ No. of Patients (yrs) } \\
\hline & $9-10$ & $10-11$ & $11-12$ & $12-13$ & $13-14$ & $14-15$ & $15-16$ & $16-17$ & $17-18$ & $18-19$ & $19-12$ & $20-21$ & $21-22$ \\
\hline good & 5 & 9 & 4 & 4 & 1 & 5 & 4 & 6 & 3 & 2 & 4 & 4 & 2 \\
\hline moderate & 0 & 0 & 0 & 0 & 0 & 0 & 0 & 0 & 0 & 0 & 0 & 0 & 0 \\
\hline poor & 0 & 0 & 0 & 0 & 0 & 0 & 0 & 0 & 0 & 0 & 0 & 0 & 0 \\
\hline death & 0 & 0 & 0 & 0 & 0 & 0 & 1 & 0 & 0 & 0 & 0 & 0 & 0 \\
\hline
\end{tabular}

\section{Procedure-Related Complications}

Postoperative bacterial infections developed in 40 patients $(30.6 \%)$ with cerebral cysticercosis who underwent surgical treatment (meningitis in 27, meningitis and pulmonary infection in four, and pulmonary and/or other infection in nine patients). In five patients $(10.2 \%)$ in whom PFC was performed, postoperative meningitis developed, and in one the wound became infected. Of the 103 procedures for VP shunt implantation, at least one episode of bacterial shunt infection occurred in 26 patients (25.2\%) during the follow-up period and in one subdural empyema occurred.

Surgery-related complications other than infections were observed in two patients (11.8\%) who underwent TCA to resect third/lateral ventricular lesions (infection of the wound in one and subdural empyema causing death in the other). In one patient (12.5\%) in whom an endoscopic approach to the ventricles was undertaken, an intraventricular hematoma developed due to venous bleeding; this led to the patient's death despite immediate open drainage.

The following neurological complications were observed in eight patients (16.3\%) who underwent PFC: CSF fistula in three, isolated fourth ventricle in two, extradural hematoma of the posterior fossa in two, and hypertensive pneumoencephalus in one. One patient with an isolated fourth ventricle, another with a posterior fossa extradural hematoma, and the one patient with hypertensive hydrocephalus died as a consequence of these complications.

Six patients $(5.7 \%)$ who underwent VP shunt surgery suffered the following postoperative complications: chronic subdural hematoma in five and hemiparesis in one.

\section{Mortality Rates}

The global mortality rate demonstrated in all patients with cerebral cysticercosis who underwent surgical treatment was $21.3 \%$ (34 patients). Surgery-related death occurred in three patients $(4.4 \%)$ in whom a reservoir was implanted, two due to ventriculitis and one to nonneurological cause. One patient who underwent a pterional craniotomy (for removal of cisternal cysts causing increased ICP) died of unknown cause early in the postoperative period. In one patient $(12.5 \%)$ who underwent removal of giant parenchymal cysts, the surgery was performed while the patient was comatose (with dilated fixed pupils), and this patient died in the early postoperative period. In one patient in whom bilateral temporal decompressive craniotomy was conducted to treat refractory increased ICP, the hypertension did not resolve and the patient died early in the postoperative period.
Of those in whom endoscopy was used to approach ventricular lesions, two patients $(25 \%)$ died early in the postoperative period, one with fourth ventricular disease due to a recurrence of a ventriculitis acquired in the preoperative period and the other with lateral ventricular disease due to an intraventricular hematoma and increased ICP.

The global mortality demonstrated in patients who underwent a TCA to cysts of the third/lateral ventricles was $17.6 \%$ (three patients). Two patients (11.8\%) died in the early postoperative course, one of pneumonia and the other of intracranial hypertension and subdural empyema. The third patient died late in the postoperative period of unknown cause.

The global mortality rate demonstrated in patients who underwent PFC was $16.3 \%$ (eight patients), and surgeryrelated death in these patients occurred in $10.2 \%$ (four early and one late surgery-related death). In the four patients in whom early surgery-related death occurred, arachnoiditis and/or ependymitis was present in the fourth ventricle/posterior fossa. The causes of death in these cases were intracranial hypertension in two patients, an extradural hematoma of the posterior fossa in one, and meningitis in the fourth. The late surgery-related death was caused by intracranial hypertension and meningitis. Three patients died of nonneurological problems.

The global and surgery-related mortality rates demonstrated in patients who underwent VP shunt surgery were $18.1 \%$ (19 patients) and $16.1 \%$ (17 patients), respectively. In these patients early and late surgery-related mortality rates were $4.8 \%$ (five patients) and $12.3 \%$ (13 patients), respectively. Causes of early death were ICP in two patients and meningitis in three patients; causes of late death were increased ICP in seven, increased ICP and meningitis/ventriculitis in five, and ventriculitis in one patient. The remaining patient died of nonneurological causes.

\section{DISCUSSION}

Our experience in the treatment of cerebral cysticercosis can be divided into two periods: before and after 1980. During the first period, the attempts at identifying the different forms and the locations of the lesions were more difficult because, in most cases, only angiography and ventriculography were available. Consequently, we had no defined protocol for the treatment of these patients, and the results of direct approaches (to the posterior fossa and supratentorial region) particularly ventricular approaches, were difficult to evaluate. Nevertheless, because shunt surgery was performed in most patients, it was possible to evaluate the long-term treatment effects in a large 
number of cases. ${ }^{17,18}$ Since 1980, CT scanning, CT cisternography, CT ventriculotomography, and, more recently, MR imaging, we have been better able to identify the pathophysiological mechanisms of these lesions, especially those causing intracranial hypertension, and to plan the best therapeutic approach in patients with cerebral cysticercosis. In addition to the advancement in neurodiagnostic modalities, the systematic use of microsurgery has contributed to improved results in selected cases.

Recent pharmacological experience with praziquantel and albendazole, which can provoke degeneration of cysticerci, $2,12,27,28,44,49,51,52,54,57,59,62$ has shown that their efficacy is more marked in cases in which active cysts are present in the brain parenchyma. Although the effect of these drugs has been reported on ventricular or cisternal cysts, ${ }^{28,44}$ there remains no consensus about their efficacy in these situations. Several of our patients with cisternal and ventricular cysts, as well as other patients with cisternal cysts (Takayanagui, personal communication) received praziquantel or albendazole in our hospital, but this treatment was not effective. Anticysticercal drugs did not prevent the evolution for intracranial hypertension in our patients. Based on the results of anticysticercal therapy in our hospital, ${ }^{57,59}$ we have prescribed albendazole in all patients with active parenchymal cysts but without signs of local compression and/or signs of intracranial hypertension or significant mass effect. In patients harboring parenchymal cysts causing local compression or giant cysts causing intracranial hypertension or significant mass effect, cyst removal is indicated. We do not recommend anticysticercal therapy for patients harboring only cisternal and/or ventricular cysts.

Among the clinical forms of NCC that can be treated surgically are 1) the spinal forms that manifest signs of spinal cord compression or of nerve root and cauda equina compression; 2) the forms that manifest signs of local compression of the brain and of cranial nerves; 3 ) and the forms that manifest intracranial hypertension.

\section{Cisternal Forms Causing Local Compression}

Patients with cisternal cysts usually harbor racemose cysticerci in the basal cisterns; the lesions are varied in size and cause symptoms of local compression independent of signs of hydrocephalus or mass effect-induced hypertension (Fig. 1). Among cranial nerves most affected by cysticerci- or arachnoiditis-induced local compression are the optic nerve and the chiasm, the oculomotor nerve, the trigeminal nerve, and the facial nerve. ${ }^{18}$ Other clinical manifestations of compression include signs related to compression of the cerebral cortex and Parinaud syndrome; ${ }^{14}$ these signs manifest secondary to compression induced by cysts located in the cistern of the quadrigeminal lamina.

Direct excision of the cysticerci in the basal cisterns is not an effective procedure. Only in rare cases can all cysts be removed because they are usually multiple, and they are frequently partially degenerated and adherent to the cranial nerves, vessels, and brain parenchyma due to arachnoiditis. An attempt at full lysis of arachnoiditis in these cases may have disastrous consequences. Resection of free cysts and even of degenerating cysts causing welldefined localized compressions, however, may be benefi- cial, as was demonstrated in several of our cases. Examples of these benefits are the stabilization or improvement of visual disorders. Nevertheless, excision of the cysts does not prevent the progression of the inflammatory reaction already underway, and good results often may be transitory.

\section{Forms Progressing to Intracranial Hypertension}

The incidence of increased ICP in patients with cerebral cysticercosis ranges from 25 to $65.9 \% .^{14,33,43,47,58}$ In our hospital hypertension was observed alone or in combination with other clinical forms in $35.7 \%$ of the patients. ${ }^{58}$ These patients could be classified in three groups according to the pathophysiological mechanism of hypertension: ${ }^{55}$ 1) hypertension caused by space-occupying cysticerci (tumoral form); 2) hypertension caused by diffuse cerebral edema (pseudotumoral form); and 3) hypertension secondary to hydrocephalus caused by obstruction of CSF circulation. These mechanisms can be isolated or combined. The relative frequency of Group 1 ranges from 22 to $67 \%$, in Group 2 from 9 to $44 \%$, and in Group 3 from 18 to $41 \%{ }^{14,55}$ In a previous series ${ }^{21}$ studied at our hospital, $2.95 \%$ of the patients who underwent surgery to control increased ICP belonged to Group 1, 5.8\% to Group 2, and $91.3 \%$ to Group 3; and in the present series, increased ICP was caused by hydrocephalus in $134(86.5 \%)$, by tumoral form in $12(7.7 \%)$, by hydrocephalus and giant cysts in eight (5.2\%), and by pseudotumor cerebri in one case. The difference demonstrated between this and our previous surgical series ${ }^{21}$ can be explained by an improvement in the clinical treatment of intracranial hypertension, thus reducing the number of cases refractory to treatment.

Because of their particularly high morbidity and mortality rates, patients afflicted with forms of NCC manifesting intracranial hypertension usually require surgical treatment.

Tumoral Form. Intracranial parenchymal (Fig. 2), ventricular, or cisternal giant cysticerci most often are racemose, ${ }^{32,39}$ and their response to anticysticercal drugs such as praziquantel and albendazole remains controversial. ${ }^{2,15,27,29,34,42,44,49,51,52,54,57,59,62}$ On the other hand, the risk of decompensation associated with increased ICP due to cyst degeneration-induced inflammatory reaction in patients with $C$. cellulosa $e^{49}$ may contraindicate drug therapy and definitively contraindicate these drugs in patients with giant cysticerci and mass effect. In our opinion, the best treatment of the tumoral form of NCC is surgicalcyst excision via a direct approach, or at least, cyst evacuation and partial resection via a direct or stereotaxic approach. Complete resection of giant cysts is usually relatively easy when the cyst is located within the parenchyma or cisterns, as well as when the cysts are in the active phase because they are still loosely adherent to the parenchyma. Degenerating cysts may adhere firmly to the nervous tissue and to blood vessels because of inflammatory reaction, especially those located in the cisterns. Resection of these cysts, especially those close to eloquent areas of the brain, is usually associated with a considerable risk of additional lesions.

Pseudotumoral or Encephalitic Form. This form is characterized by increased ICP secondary to a diffuse inflammatory reaction of the brain parenchyma due to mas- 
sive infestation with cisticerci, $, 8,14,55$ and it is more frequent in children. ${ }^{14,36,55}$ Treatment of this form is primarily medical to reduce the intracranial hypertension by administering osmotic diuretics, steroid agents, and by performing CSF lumbar puncture, and it is effective in most cases. ${ }^{8,13}$ In the past, decompressive craniectomy was recommend$\mathrm{ed}^{8}$ in these patients to avoid visual loss due to papilledema, but its results were only transient. ${ }^{55}$ In our opinion decompressive craniotomy is only rarely indicated and should be performed in extreme situations in an attempt to control intracranial hypertension. Fortunately, the decrease in the number of decompressive craniotomy-treated patients between our previous surgical series ${ }^{21}$ and this series, suggests an improvement in the clinical treatment of intracranial hypertension. Although there are no published data about the use of lumboperitoneal shunts for treating these patients, it does not appear to be a good solution because the intracranial hypertension in this process is caused more by an increased volume of the brain parenchyma than by an increase in CSF volume.

Obstructive Form. Hydrocephalus is the most frequent mechanism of intracranial hypertension in NCC, and it can be caused by mechanical obstruction of the ventricles or the basal cisterns. Obstruction of the former is caused either by cysts themselves or by the inflammatory reaction (ependymitis, arachnoiditis, or both) or may be caused by difficulties in absorbing CSF due to parasagittal arachnoiditis, with involvement of arachnoid villi. ${ }^{36,37,46,50,55}$ Cyst-related obstruction usually occurs at the narrow sites of the ventricular system (for examples, the foramen of Magendie and the cerebral aqueduct), causing obstructive hydrocephalus. Cysts localized in the basal cisterns can provoke communicating hydrocephalus. Inflammatory reaction-related obstruction is more frequent in the outgoing foramina of the fourth ventricle, the posterior fossa, and the basal cisterns, but it may occur at any point in the CSF circulation, including sites of CSF absorption, and cause obstructive or communicating hydrocephalus.

Rational treatment of cysticercosis-related hydrocephalus requires identification of its pathogenesis. Ventriculography is simple, easy to perform, and accurate for this purpose. Based on ventriculographic findings, we have sought to identify the site and type of obstruction ${ }^{18}$ - that is, in patients with obstruction caused by free cysts who can benefit from cyst removal, $, 17,31,38,40,50,56$ or in patients with obstruction caused by an inflammatory process in whom VP shunt surgery is considered the best treatment. ${ }^{3,16,33,35,38}$ Generally CT scanning does not allow this differentiation because it does not reveal free intraventricular cysts, ${ }^{19}$ which are only be suspected based on the morphological changes of the ventricles (rounding of the fourth or third ventricles or of portions of the lateral ventricles). Computerized tomography cisternography demonstrates cysts in the cisterns; additionally, when there is no obstruction of the ventricular system, it identifies intraventricular cysts (Figs. 3 and 5). Computerized tomography ventriculography allows adequate evaluation of the ventricular system, including the identification of cysts not visualized on ventriculography alone. ${ }^{18,39}$ Magnetic resonance imaging is useful because it can demonstrate cysticerci and inflammatory reactions in the parenchyma, cisterns, and even ventricles (Fig. 4). Unless there are in- direct signs, however, such as mass effect, absence of the adjacent CSF flow, and obstruction of the ventricular flow, intraventricular cysts are visualized on MR images only after they start to degenerate. ${ }^{41,60}$

In our service a ventricular reservoir has been routinely implanted in patients with acquired hydrocephalus to perform CT ventriculography and for relief of intracranial hypertension, until definitive diagnostic and therapeutic procedures can be undertaken. By following this protocol, ventricular cysts can be excised, and the decision regarding definitive CSF shunt placement can be postponed, depending on the recurrence of increased ICP. More recently, since MR imaging became available at our institution, this neurodiagnostic modality has allowed identification of intraventricular cysts in some patients (Fig. 4), thus obviating need for reservoir implantation.

Excision of free ventricular cysts is relatively simple when performed via a suitable approach and when using microsurgical techniques; in such cases morbidity and mortality rates are minimal. This procedure is justified because free cysts may dislocate within the ventricular system, ${ }^{21}$ causing acute intracranial hypertension by obstructing CSF circulation, and because intraventricular cysts tend to increase in size and start to behave as intraventricular tumors, especially in the fourth ventricle (Fig. 5). Access to the fourth ventricle is obtained by posterior fossa craniotomy, and the free cysts can spontaneously protrude through the foramen of Magendie toward the cistern magna ("delivery" of the cysticercus) (Figs. 3 and 5), or they may be tractioned. When the cysticerci are adherent to the wall of the ventricle or when the foramen of Magendie is stenotic or obstructed, the foramen needs to be opened or widened by sectioning the inferior portion of the cerebellar vermis to allow better visualization of the ventricle.

Free cysts located in the lateral ventricle or in the third ventricle can be reached via an anterior TCA. We recommend a direct approach and extirpation of the lateral ventricular cyst when it is free and at risk of dislocating; when the cyst is adherent to the ventricle walls, we perform excision only if it causes septations of the cavities and/or obstruction of CSF circulation. A temporal horn cyst is better reached via a temporal transcortical approach.

The mortality rate observed in patients who have undergone PFC range from 0 to $60 \%$. $^{6,17,18,37,38,40,43,56}$ The lowest mortality rates have been demonstrated in cases in which those who present with free ventricular cysts, and the highest rates have been shown in those who present with inflammation-related obstruction or cyst- and inflammation-related obstructions. For this reason, we try to identify patients with free ventricular cysts and recommend a direct-approach resection only in these cases. The high mortality and morbidity rates observed in the past among patients who have undergone PFC were related to the difficulties in the surgical management of patients with arachnoiditis and aseptic meningitis caused by intraoperative cyst rupture. Although these rates have been reduced (by better identification of patients with free cysts, microsurgical techniques, and by the satisfactory control of aseptic meningitis with steroids ${ }^{21}$ ), analysis of our recent long-term follow-up data has shown that the morbidity and mortality rates remain high. A direct surgical approach is indicated with reservation when radiological ex- 


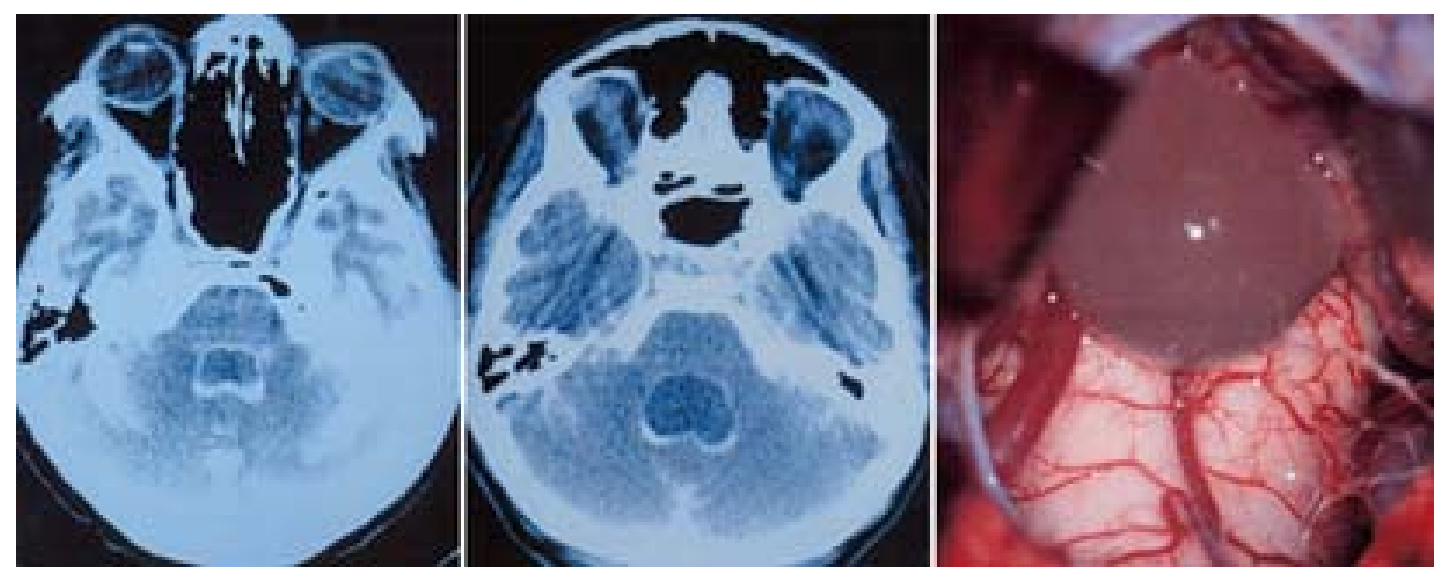

Fig. 5. Left: A CT cisternography scan obtained in a patient with intermittent increased ICP, demonstrating a cyst in the fourth ventricle delineated by contrast. The patient refused surgical treatment at that time and returned 1 year later with worsened symptoms and severe gait ataxia. Center: Repeated CT cisternography revealing an increase in the size of the cyst. Right: Intraoperative photograph of the posterior fossa obtained with the patient in the sitting position. After gentle lateral displacement of the cerebellar tonsils, the cyst is spontaneously protruding from the ventricle ("delivery" of the cysticercus). Note that the cyst membrane is transparent and that its fluid content is colorless and that there is no arachnoiditis in the posterior fossa.

aminations do not allow differentiation between inflammation- and cyst-related obstructions, taking into consideration the advantages and disadvantages of a ventricular approach.

Other procedures such as a cystoperitoneal shunt surgery, ${ }^{7}$ stereotactic approaches to cyst resection or drainage, ${ }^{24,48,61}$ and endoscopic cyst drainage or resection ${ }^{4,10,11,25}$ have been proposed as alternatives to extirpation of giant parenchymal and ventricular cysts. Cyst evacuation through puncturing may be effective because, theoretically, the rupture of a cyst triggers its process of degeneration. Cyst recurrence after puncture and drainage ${ }^{24,56}$ should be attributed to the existence of racemose cyst at the punctured site. We prefer undertaking the direct surgical approach in patients with giant cysts because it is relatively safe when the appropriate surgical strategy is used, it allows better evaluation of the adherence of cysts in relation to adjacent structures, and it permits the resection or emptying of the various vesicles of a racemose cysticercus; additionally analysis of our previous and recent data has shown that it is efficient in most patients. ${ }^{17,18} \mathrm{~A}$ direct approach to free ventricular cysts is our first option as well because it is possible to identify the presence of ependymitis/arachnoiditis. When inflammatory reactions are intense, we perform VP shunt surgery immediately after the cyst is resected, because in our previous and recent experience these patients usually develop inflammatory blockade of the CSF circulation and intracranial hypertension within a few weeks or months. ${ }^{17,18}$ Recently we began to perform endoscopic approaches to the ventricles for cyst removal, and we have encountered some complications, including death, probably as a result of technical problems and incorrect selection of cases. Nevertheless, because it is less invasive, and because manipulation under direct visualization and identification of multiple vesicles are possible, the endoscopic approach may become the procedure of choice in many of these patients.
Most of our patients with inflammatory obstruction of CSF circulation underwent VP shunt placement because previous experience ${ }^{6,17,18,37,45,46}$ has shown that these patients do not benefit from a direct approach to the posterior fossa for lysis of the inflammatory reaction, which recurs within a short time (generally a few weeks), and to avoid serious complications. ${ }^{17,18}$

The placement of a VP shunt has been considered the best treatment in patients with hydrocephalus due to inflammatory obstruction caused by cysticercosis, allowing for resolution of increased ICP in 50 to $90 \%$ of cases. Our results confirm the previous experience; most patients were free from hypertension at the end of a 2-year followup period, and more than half required revisions of the shunt, most during the first postoperative year. ${ }^{16,17,21,43}$ The predominance of complications in this period probably occurs because most cysts of a multiple infestation degenerate within that time. Another possibility is that within the follow-up period functional equilibrium in the CSF circulation is reached, and the shunt, which may not be functioning, is no longer required. Isolation of the fourth ventricle has been reported in patients with cerebral cysticercosis, generally related to a VP shunt. ${ }^{22,23,26}$ This is usually a serious complication, and details of this entity have been discussed in a previous study. ${ }^{22}$

Some authors ${ }^{31,37,39}$ recommend that VP shunt placement should be performed as the first surgical procedure in all patients with hydrocephalus secondary to cysticercosis to relieve intracranial hypertension and that ventricular cysts should be removed during a second stage. Currently we prefer first to control intracranial hypertension by implanting a ventricular reservoir and determining in advance whether the patient will benefit from the removal of a free cyst or whether VP shunt surgery will be the best treatment. This strategy has been adopted because of the risks associated with technical difficulties involved in approaching a fourth ventricle in which there is ependymitis and which has collapsed after placement of a VP shunt, as 
well as to ensure that some patients will not receive an unnecessary shunt.

\section{Prognostic Factors}

In patients with cerebral cysticercosis the location of cysts in the subarachnoid space (extraparenchymal cysts), especially at the basal cisterns, causing chronic arachnoiditis, has been identified as poor prognostic factor ${ }^{9,17,18,50}$ and this was confirmed by our data. In patients requiring VP shunt surgery for the treatment of inflammatory obstruction or inflammatory obstruction and cysts, the prognosis was worse than in those who underwent removal of intraventricular cysts. This is because these patients require a shunt and its incidence of associated complications is higher than in patients with hydrocephalus than with other diseases. ${ }^{16}$

Age was also found to be a prognostic factor in patients requiring surgical treatment. Prognosis was poorer in patients younger than 40 years of age than in those who were older. Some authors ${ }^{50}$ have found the incidence of the extraparenchymal form of the cerebral cysticercosis to be higher in females, and this could be related to a poor prog- nosis. Analysis of the survival curves according to sex for all patients and for patients who underwent reservoir/ shunt implantation and shunt implantation alone showed a poor prognosis for males. Satisfactory explanations for these facts were not found.

\section{CONCLUSIONS}

In patients with cerebral cysticercosis requiring surgery, the long-term prognosis was not good. Overall 1.75 surgical procedures per patient were performed in these cases, and there were many surgery-related complications. The global mortality rate during the follow-up period and the surgery-related mortality rate were also high. Location of cysts in basal cisterns, age younger than 40 years, and male sex were related to worse prognosis. In patients with inflammatory process or cysts combined with an inflammatory process requiring placement of a VP shunt and in patients requiring surgery who were younger than 40 years of age prognosis was worse than in other patients.

The diagrams in Fig. 6 depict our recent policy in the treatment of patients with CNS cysticercosis.

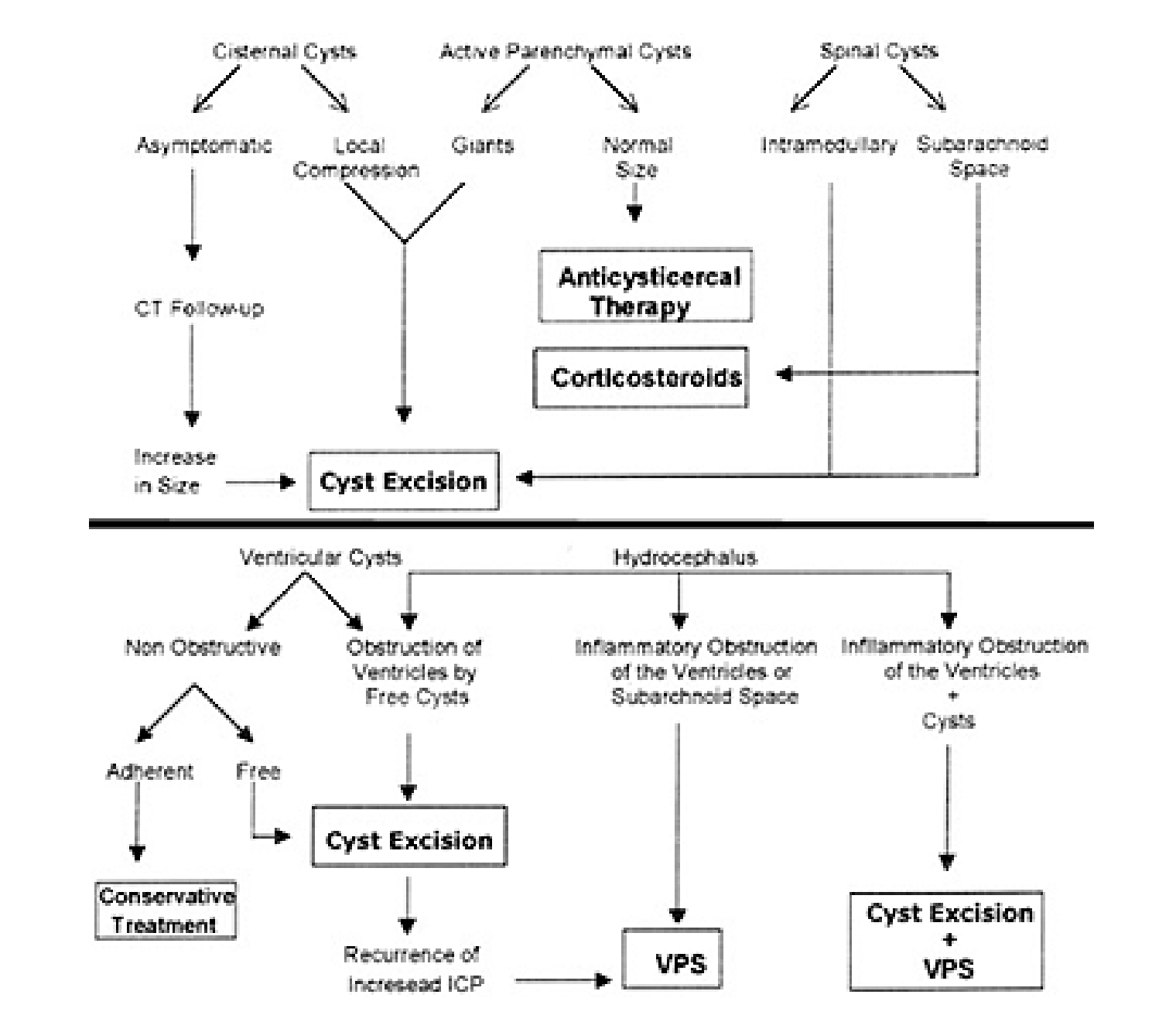

Fig. 6. Upper: Diagram depicting management of patients with parenchymal and cisternal NCC. Lower: Diagram depicting management of patients with intraventricular cysts and hydrocephalus due to cerebral cysticercosis. VPS = VP shunt. 


\section{References}

1. Agapejev S: Epidemiology of neurocysticercosis in Brazil. Rev Inst Med Trop Sao Paulo 38:207-216, 1996

2. Agapejev S, Meira DA, Barraviera B, et al: Neurocysticercosis: treatment with albendazole and dextrochloropheniramine (preliminary report). Rev Inst Med Trop Sao Paulo 30:387-389, 1988

3. Almeida GM, Pereira WC, Facure NO: Ventriculo-auriculostomia nos bloqueios ao trânsito do líquido cefalorraqueano na cisticercose encefálica. Arq Neuropsiquiatr 24:163-168, 1966

4. Anandh B, Mohanty A, Sampath S, et al: Endoscopic approach to intraventricular cysticercal lesions. Minim Invasive Neurosurg 44:194-196, 2001

5. Apuzzo ML, Dobkin WR, Zee CS, et al: Surgical consideration in treatment of intraventricular cysticercosis. An analysis of 45 cases. J Neurosurg 60:400-407, 1984

6. Arana R, Asenjo A: Ventriculographic diagnosis of cysticercosis of the posterior fossa. J Neurosurg 2:181-190, 1945

7. Araújo LP, Martelli N, Marquez JO: Forma cistica gigante da neurocisticercose: relato de caso. Arq Bras Neurocir 3: 119-123, 1984

8. Arseni C, Samtica DC: Cysticercosis of the brain. Br Med J 2: 494-497, 1957

9. Bandres JC, White AC Jr, Samo T, et al: Extraparenchymal neurocysticercosis: report of five cases and review of management. Clin Infect Dis 15:799-811, 1992

10. Bergsneider M: Endoscopic removal of cysticercal cysts within the fourth ventricle. Technical note. J Neurosurg 91:340-345, 1999

11. Bergsneider M, Holly LT, Lee JH, et al: Endoscopic management of cysticercal cysts within the lateral and third ventricles. J Neurosurg 92:14-23, 2000

12. Botero D, Uribe CS, Sanchez JL, et al: Short course albendazole treatment for neurocysticercosis in Columbia. Trans R Soc Trop Med Hyg 87:576-577, 1993

13. Braga FM, Ferraz FA: Forma edematosa da neurocisticercose. Arq Neuropsiquiatr 39:434-443, 1981

14. Canelas HM: Neurocisticercose: incidência, diagnóstico e formas clínicas. Arq Neuropsiquiatr 20:1-16, 1962

15. Carpio A, Santillan F, Leon P, et al: Is the course of neurocysticercosis modified by treatment with antihelminthic agents? Arch Intern Med 155:1982-1988, 1995

16. Colli BO: Contribución al estudio del tratamiento quirúrgico de la neurocysticercosis. Gac Med Mex 117:251-257, 1981

17. Colli BO, Martelli N, Assirati JA Jr, et al: Results of surgical treatment of neurocysticercosis in 69 cases. J Neurosurg 65: 309-315, 1986

18. Colli BO, Martelli N, Assirati Junior JA, et al: Cysticercosis of the central nervous system. I. Surgical treatment of cerebral cysticercosis: a 23 years experience in the Hospital das Clinicas of Ribeirao Preto Medical School. Arq Neuropsiquiatr 52: 166-186, 1994

19. Colli BO, Martelli N, Assirati Junior JA, et al: Forma tumoral da neurocisticercose: exérese de cisticerco de $70 X 77 \mathrm{~mm}$ e tratamento com praziquantel: relato de caso. Arq Neuropsiquiatr 42:158-165, 1984

20. Colli BO, Martelli N, Assirati Junior JA, et al: Tomografia computadorizada em pacientes portadores de neurocisticercose com hipertensão intracraniana por hidrocefalia obstrutiva: comparação com ventriculografia com Dimer-X. Arq Neuropsiquiatr 42:116-125, 1984

21. Colli BO, Martelli N, Assirati Junior JA, et al: Ventriculografia com Dimer-X em pacientes portadores de neurocisticercose com hipertensão intracraniana por hidrocefalia obstrutiva. Arq Bras Neurocir 2:197-206, 1983

22. Colli BO, Pereira CU, Assirati Junior JA, et al: Isolated fourth ventricle in neurocysticercosis: pathophysiology, diagnosis, and treatment. Surg Neurol 39:305-310, 1993
23. Costa V, Costa J, Portela LAP: IV ventrículo isolado: considerações e relato de 3 casos. Arq Bras Neurocir 4:123-134, 1985

24. Couldwell WT, Zee CS, Apuzzo ML: Definition of the role of contemporary surgical management in cisternal and parenchymatous cysticercosis cerebri. Neurosurgery 28:231-237, 1991

25. Cudlip SA, Wilkins PR, Marsh HT: Endoscopic removal of a third ventricular cysticercal cyst. Br J Neurosurg 12:452-454, 1998

26. DeFeo D, Foltz EL, Hamilton AE: Double compartment hydrocephalus in a patient with cysticercosis meningitis. Surg Neurol 4:247-251, 1975

27. del Brutto OH, Sotelo J: Albendazole therapy for subarachnoid and ventricular cysticercosis. Case report. J Neurosurg 72: 816-817, 1990

28. Del Brutto OH, Sotelo J, Aguirre R, et al: Albendazole therapy for giant subarachnoid cysticerci. Arch Neurol 49:535-538, 1992

29. Del Brutto OH, Sotelo J, Roman GC: Therapy for neurocysticercosis: a reappraisal. Clin Infect Dis 17:730-735, 1993

30. Escobar A: The pathology of neurocysticercosis, in Palacios E, Rodriguez-Carbajal J, Taveras JM (eds): Cysticercosis of the Central Nervous System. Springfield, IL: Charles C Thomas, 1983, pp 27-54

31. Estañol B, Kleriga E, Loyo M, et al: Mechanisms of hydrocephalus in cerebral cysticercosis: implications for therapy. Neurosurgery 13:119-123, 1983

32. Facure NO, Facure JJ, Nucci A: Aspecto tumoral da cisticercose intracraniana: abordagem cirúrgica. Arq Neuropsiquiatr 36:200-209, 1978

33. Grisolia JS, Wiederholt WC: CNS cysticercosis. Arch Neurol 39:540-544, 1982

34. Gupta R, Yadav NV: Failure of praziquantel and albendazole in neurocysticercosis. J Assoc Physicians India 38:313-314, 1990

35. Lobato RD, Lamas E, Portillo JM, et al: Hydrocephalus in cerebral cysticercosis. Pathogenic and therapeutic considerations. J Neurosurg 55:786-793, 1981

36. Lombardo L, Mateos JH, Estañol B: La cysticercosis cerebral en México. Gac Med Mex 118:1-16, 1982

37. Loyo M, Kleriga E, Estañol B: Fourth ventricular cysticercosis. Neurosurgery 7:456-458, 1980

38. Macías Sanchez R, Hernandez Peniche J: Cisticercosis cerebral: diagnóstico clínico, radiológico y de laboratório, pronóstico. Prensa Med Mex 31:147-155, 1966

39. Madrazo I, García-Rentería JA, Paredes G, et al: Diagnosis of intraventricular and cisternal cysticercosis by computerized tomography with positive intraventricular contrast medium. J Neurosurg 55:947-951, 1981

40. Madrazo I, Garcia-Renteria JA, Sandoval M, et al: Intraventricular cysticercosis. Neurosurgery 12:148-152, 1983

41. Mancuso P, Guarnera F, Augello G, et al: Computed axial tomography versus NMR for the diagnosis of neurocysticercosis. Neurochirurgia 30:152-153, 1987

42. Martinez HR, Rangel-Guerra R, Arredondo-Estrada JH, et al: Medical and surgical treatment in neurocysticercosis a magnetic resonance study of 161 cases. J Neurol Sci 130:25-34, 1995

43. McCormick GF, Zee CS, Heiden J: Cysticercosis cerebri. Review of 127 cases. Arch Neurol 39:534-539, 1982

44. Proano JV, Madrazo I, Avelar F, et al: Medical treatment for neurocysticercosis characterized by giant subarachnoid cysts. $\mathbf{N}$ Engl J Med 345:879-885, 2001

45. Pupo PP, Pimenta AM: Cisticercose do IV ventrículo. Considerações anátomo-clinicas e sobre a terapêutica cirúrgica. Arq Neuropsiquiatr 7:274-290, 1949

46. Salazar A, Sotelo J, Martinez H, et al: Differential diagnosis between ventriculitis and fourth ventricle cyst in neurocysticercosis. J Neurosurg 59:660-663, 1983

47. Santos JC, Balbo RJ, Sarian L, et al: Neurocisticercose: aspec- 


\section{Surgical treatment of cerebral cysticercosis}

tos clínicos e cirúrgicos. Estudo de 205 casos. Arq Bras Neurocir 7:203-210, 1988

48. Seigel RS, Davis LE, Kaplan RJ, et al: CT-guided aspiration of a cysticercotic thalamic cyst. Bull Clin Neurosci 48:48-60, 1983

49. Sotelo J, del Bruto OH, Penagos P, et al: Comparison of therapeutic regimen of anticysticercal drugs for parenchymal brain cysticercosis. J Neurol 237:69-72, 1990

50. Sotelo J, Marin C: Hydrocephalus secondary to cysticercosis arachnoiditis. A long-term follow-up review of 92 cases. J Neurosurg 66:686-689, 1987

51. Sotelo J, Penagos P, Escobedo F, et al: Short course of albendazole therapy for neurocysticercosis. Arch Neurol 45: 1130-1133, 1988

52. Sotelo J, Torres B, Rubio-Donnadieu F, et al: Praziquantel in the treatment of neurocysticercosis: long-term follow-up. Neurology 35:752-755, 1985

53. Spina-França A: Neurocisticercose e imunobiologia. J Bras Med 45 (Suppl):7-8, 1983

54. Spina-Franca A, Nobrega JP, Machado LR, et al: [Neurocysticercosis and praziquantel: long-term development in $100 \mathrm{pa}-$ tients.] Arq Neuropsiquiatr 47:444-448, 1989 (Por)

55. Stepién L, Choróbski J: Cysticercosis cerebri and its operative treatment. Arch Neurol Psychiatry 61:499-527, 1949

56. Stern WE: Neurosurgical considerations of cysticercosis of the central nervous system. J Neurosurg 55:382-389, 1981
57. Takayanagui OM: Neurocisticercose. II. Avaliação da terapêutica com praziquantel. Arq Neuropsiquiatr 48:11-15, 1990

58. Takayanagui OM, Jardim E: Aspectos clínicos da neurocisticercose: análise de 500 casos. Arq Neuropsiquiatr 41:50-63, 1983

59. Takayanagui OM, Jardim E: Therapy for neurocysticercosis. Comparison between albendazole and praziquantel. Arch Neurol 49:290-294, 1992

60. Teitelbaum GP, Otto RJ, Lin M, et al: MR imaging of neurocysticercosis. AJR 153:857-866, 1989

61. Teixeira MJ, Nóbrega JPS, Oliveira JO Jr, et al: Exérese estereotomográfica de cistos intracranianos na cisticercose. Arq Bras Neurocir 7:221-223, 1988

62. Vasconcelos D, Cruz-Segura H, Mateos-Gomes H, et al: Selective indications for the use of praziquantel in the treatment of of brain cysticercosis. J Neurol Neurosurg Psychiatry 50: 383-388, 1987

Manuscript received April 15, 2002.

Accepted in final form June 15, 2002.

Address reprint requests to: Benedicto Oscar Colli, M.D., Departamento de Cirurgia, HCFMRP, Campus Universitário USP, 14048-900 Ribeirão Preto, Sao Paulo, Brazil. email: bocolli@ fmrp.usp.br. 